\title{
Matched Filtering for Generalized Stationary
}

\section{Processes}

\author{
Vadim Olshevsky and Lev Sakhnovich \\ Department of Mathematics \\ University of Connecticut \\ Storrs, CT 06269
}

This work was supported in part by the NSF contracts 0242518 and 0098222.

web page: http://www.math.uconn.edu/ ${ }^{\circ}$ olshevsky

email: olshevsky@math.uconn.edu 


\begin{abstract}
The methods for solving optimal filtering problems in the case of the classical stationary processes are well-known since the late forties. Practice often gives rise to what is not a classical stationary process but a generalized one, and white noise is one simplest example. Hence it is of interest to describe the system action on the generalized stationary processes, and then to carry over filtering methods to them. For the arbitrary generalized stochastic processes this seems to be a challenging problem. In this correspondence we identify a rather general class of $S_{J}$-generalized stationary processes for which the desired extension can be done for matched filters. This class can be considered as a model of colored noise, and it is wide enough to include white noise, positive frequencies white noise, as well as certain generalized processes occurring in practice, namely when the smoothing effect gives rise the situation in which the distribution of probabilities may not exist at some time instances. One advantage of the suggested model is that it connects optimal filter design with inverting of integral operators; the methods for the latter can be found in the extensive literature.
\end{abstract}

\title{
I. Motivation
}

1.1. Classical Stationary Processes. A stochastic process $x(t)$ is called stationary in the wide sense if its expectation is a constant,

$$
E[x(t)]=\text { const },
$$

and the correlation function depends only on the difference $(t-s)$, i.e.,

$$
E[x(t) \overline{x(s)}]=K_{x}(t-s) .
$$

We assume that $E\left[|x(t)|^{2}\right]<\infty$.

Let us consider a system with the memory depth of $\omega$ that maps the input stochastic process $x(t)$ into the output stochastic process $y(t)$ in accordance with the following rule:

$$
y(t)=\int_{t-\omega}^{t} x(s) h(t-s) d s .
$$

Wiener's seminal monograph [W49] described the construction of the optimal filter in the case $\omega=\infty$. His results were extended to the case $\omega<\infty$ in [ZR50].

1.2. Wiener filters and matched filters. Let the system receive a signal $a(t)$ corrupted by noise $x(t)$, which we assume, for a moment, to be a zero-mean stochastic process stationary in the wide sense (the more natural generalized stationary case is discussed below). In accordance with (1) the system output is

$$
a_{o}(t)+y(t)=\int_{0}^{T} h(\tau)[a(t-\tau)+x(t-\tau)] d \tau,
$$


as shown in the following picture

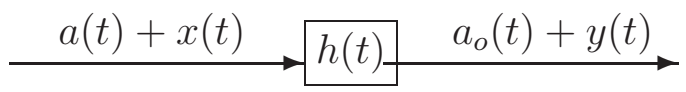

Figure 1. Classical Optimal Filter.

The objective is to find the form of $h(t)$ that makes the system optimal in the sense of a certain criterion. Two particular criteria are recalled next.

Wiener filter. In the case of random signals the typical criterion is to minimize the meansquare value of the difference between the system output $a_{o}(t)+y(t)$ and the actual value $a(t)$ being observed. In this case $h(t)$ is called the Wiener filter [H00].

Matched filter. Here we consider a different approach mostly used when the signal is deterministic. In this case the standard criterion is to maximize the signal-to-noise ratio

$$
S / N=\frac{a_{o}^{2}\left(t_{0}\right)}{\sigma^{2}}, \quad \text { where } \quad \sigma^{2}=E\left[|y(t)|^{2}\right]=\int_{0}^{T} \int_{0}^{T} h(u) B(u-v) h(v) d v d u,
$$

where $B(u)$ is the correlation function of the process $x(t)$. Such filters are referred to as matched filters [H00]. Matched filters are heavily used, e.g., in radar systems [LM04]. Radar systems operate by periodically transmitting very short bursts of radio-frequency energy. The received signal is simply one or more replicas of the transmitted signal that are created by being reflected from any objects that the transmitted signal illuminates. Thus, the form of the received signals is known exactly. The things that are not known about received signals are the number of reflections, the time delay between the transmitted and received signals, the amplitude, and even whether there is a received signal or not. It can be shown that the probability of detecting a weak radar signal in the presence of noise is greatest when the signal-to-noise ratio is the greatest.

A similar situation arises in digital communication systems [H00]. In such a system the message to be transmitted is converted to a sequence of binary signals, say 0 and 1 . Each of them is represented as a time function having a specified form. For example, a negative rectangular pulse can represent 0 while a positive rectangular pulse can represent 1. Again, in the presence of noise the probability to recognize the right pulse at the receiver is greatest when the signal-tonoise ratio is greatest.

1.3. An extension to generalized stationary processes. Motivation. The white noise $x(t)$ (having equal intensity at all frequencies within a broad band) is not a stochastic process in the classical sense as defined above. In fact, white noise can be thought of as the derivative of a Brownian motion, which is a continuous stationary stochastic process $W(t)$. It can be shown that 
$W(t)$ is nowhere differentiable, a fact explaining the highly irregular motions that Robert Brown observed. This means that white noise $\frac{d W(t)}{d t}$ does not exist in the ordinary sense. In fact, white noise is a generalized stochastic process (defined, e.g., in the main text below), cf. with [VG61].

Generally, any receiving device has a certain "inertia" and hence instead of actually measuring the classical stochastic process $\xi(t)$ it measures its averaged value

$$
\Phi(\varphi)=\int \varphi(t) \xi(t) d t
$$

where $\varphi(t)$ is a certain function characterizing the device. Small changes in $\varphi$ yield small changes in $\Phi(\varphi)$ (small changes in the receiving devices yield closer measurements), hence $\Phi$ is a continuous linear functional (see (3)), i.e., a generalized stochastic process [VG61].

Hence it is very natural and important to solve the optimal filtering problem in the case of generalized stochastic processes.

1.4. The main result and the structure of the correspondence. In Sec. II we recall the definition of generalized stationary processes, and consider a special class that contains white noise as well as certain other naturally occurring subclasses.

A disadvantage of the formulas (1) and (2) is that they are valid for the classical stationary processes only. In Sec. III we specify one way to extend these formulas to describe the system action on the generalized stationary processes, e.g., on white noise.

In Sec. IV we introduce the the concept of generalized optimal filters that operate on the generalized stationary processes. We prove that in the simplest case of classical stationary processes our definition coincides with the standard one. We then formulate the problem of designing the generalized matched filter.

In Sec. $\mathrm{V}$ we consider a special class of the so called $S_{J}$-generalized processes $\Phi$, i.e., such that for the subclass of $\varphi(t), \psi(t)$ that vanish on $J=[a, b]$ we have

$$
E[\Phi(\varphi) \Phi(\psi)]=\left(S_{J} \varphi, \psi\right)_{L^{2}}, \quad \text { where } S_{J} \varphi=\frac{d}{d t} \int_{a}^{b} \varphi(u) s(t-u) d u \in L^{2}(a, b) .
$$

We provide formulas that completely solve the problem of designing $S_{J}$-generalized matched filters. We show that if the equation $S_{J} f_{0}=g_{0}$, with $\quad g_{0}(t)=a\left(t_{0}-t\right)$. is solvable then the matched filter is given by $h_{\text {opt }}=\frac{f_{0}}{\left(g_{0}, f_{0}\right)_{L^{2}}}$.

Finally, in Sec. VI we discuss the practical consequences of the obtained result. In general, the operator $S_{J}$ can be considered as a new model to study colored noise. The suggested technique can be useful for two reasons. First, in the generalized case the operator $S_{J}$ is often invertible (in 
the classical case it is often not invertible), and hence inverting it leads to closed form solutions or to efficient algorithms to compute them. Secondly, the existing integral equations literature offers a variety of methods to invert $S_{J}$. We provide two examples indicating the above two advantages.

\section{Generalized Stationary Processes}

Let $\mathcal{K}$ be the set of all infinitely differentiable finite functions. A stochastic functional $\Phi$ assigns to any $\varphi(t) \in \mathcal{K}$ a stochastic value $\Phi(\varphi)$. A stochastic functional $\Phi$ is called linear if

$$
\Phi(\alpha \varphi+\beta \psi)=\alpha \Phi(\varphi)+\beta \Phi(\psi) .
$$

Let us now assume that all the stochastic values $\Phi(\varphi)$ have expectations $m(\varphi)$ that depend continuously on $\varphi$ as

$$
m(\varphi)=E[\Phi(\varphi)]=\int_{-\infty}^{\infty} x d F(x), \quad \text { where } \quad F(x)=P[\Phi(\varphi) \leq x] .
$$

The functional $m(\varphi)$ is linear in the space $\mathcal{K}$. The bilinear functional

$$
B(\varphi, \psi)=E[\Phi(\varphi) \overline{\Phi(\psi)}]
$$

is a correlation functional of a stochastic process. It is supposed that that $B(\varphi, \psi)$ is continuously dependent on each of the arguments.

The stochastic process $\Phi$ is called generalized stationary in the wide sense [VG61], [S97] if for any functions $\varphi(t)$ and $\psi(t)$ from $\mathcal{K}$ and for any number $h$ the equalities

$$
\begin{gathered}
m[\varphi(t)]=m[\varphi(t+h)] \\
B[\varphi(t), \psi(t)]=B[\varphi(t+h), \psi(t+h)]
\end{gathered}
$$

hold true.

Let us denote by $\mathcal{K}_{J}$ the set of the functions from $\mathcal{K}$ such that $\varphi(t)=0$ when $t \notin J=[a, b]$. The correlation functional $B_{J}(\varphi, \psi)$ is called a segment of the correlation functional $B(\varphi, \psi)$ if

$$
B_{J}(\varphi, \psi)=B(\varphi, \psi), \quad \varphi, \psi \in \mathcal{K}_{y}
$$

In what follows we consider the generalized stationary processes of the form

$$
B_{J}(\varphi, \psi)=\left(S_{J} \varphi, \psi\right)_{L^{2}}
$$


where $(\cdot, \cdot)_{L^{2}}$ is the inner product in the space $L^{2}(a, b)$, and $S_{J}$ is a bounded nonnegative operator acting in $L^{2}(a, b)$ and having the form

$$
S_{J} \varphi=\frac{d}{d t} \int_{a}^{b} \varphi(u) s(t-u) d u .
$$

Definition 1: Generalized stationary processes satisfying (7) and (8) are called $S_{J}$-generalized processes.

Example 1: White noise. It is well-known [L68] that the correlation functional for the Wiener stochastic process $\Upsilon$ (Brownian motion) is given by

$$
B(\varphi, \psi)=\int_{0}^{\infty}[\widehat{\varphi}(t)-\widehat{\varphi}(\infty)] \overline{[\hat{\psi}(t)-\widehat{\psi}(\infty)]} d t
$$

where

$$
\widehat{\varphi}(t)=\int_{0}^{t} \varphi(t) d t, \quad \widehat{\psi}(t)=\int_{0}^{t} \psi(t) d t .
$$

White noise $W$ (which is the derivative of $\Upsilon$ ) is not a continuous stochastic process. In fact, it is a generalized stationary process whose correlation functional is known [L68] to be

$$
B^{\prime}(\varphi, \psi)=\int_{0}^{\infty} \int_{0}^{\infty} \delta(t-s) \varphi(t) \overline{\psi(s)} d s
$$

Thus, in this case we have $B^{\prime}(\varphi, \psi)=(\varphi, \psi)_{L_{2}}$ and hence (7) implies that white noise $\Phi$ is a very special $S_{J}$-generalized stationary process with

$$
S_{J}=I
$$

\section{SYSTEM ACTION ON THE GENERALIZED STATIONARY PROCESSES}

Let the system receives the generalized stationary signal $\Phi$ and the deterministic signal $a(t)$. We assume that $\Phi$ is zero-mean and the correlation functional $B(\varphi, \psi)$ is known. At the output we obtain the generalized process of the form

$$
a_{o}(t)+\Psi
$$

where

$$
a_{o}(t)=\int_{0}^{T} h(\tau) a(t-\tau) d \tau
$$

cf. with (1) and (2).

The problem is how to describe the system action for the generalized stationary processes shown in the Figure 2. 


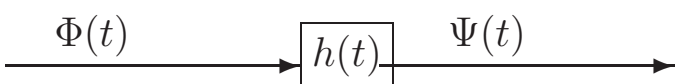

Figure 2. Generalized stationary processes

Here we answer the latter question and define it as follows

$$
\Psi(\varphi)=\Phi\left[\int_{0}^{T} h(\tau) \varphi(t+\tau) d \tau\right]
$$

so that the overall system is described in Figure 3.

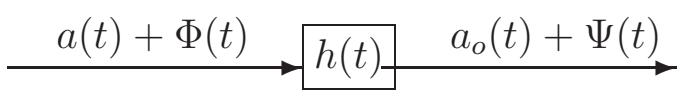

Figure 3. Generalized Matched Filter.

Proposition 1: Let $x(t)$ and $y(t)$ be the classical stationary processes. Then the formula (12) is equivalent to the relation

$$
y(t)=\int_{0}^{T} x(t-\tau) h(\tau) d \tau .
$$

Remark 1: The proposition 1 has the following meaning. The formula (13) describes the behavior of a a classical system, cf. with (1) and (2). It follows that the formula (12) suggested here indeed generalizes (13). In the case of the classical stationary processes our definition coincides with the standard one.

Proof of the proposition 1. The functionals

$$
\Phi(\varphi)=\int_{-\infty}^{\infty} x(t) \varphi(t) d t
$$

and

$$
\Psi(\varphi)=\int_{-\infty}^{\infty} y(t) \varphi(t) d t
$$

are associated with the processes $x(t)$ and $y(t)$. It follows from (13), (15) that

$$
\begin{gathered}
\Psi(\varphi)=\int_{-\infty}^{\infty} \int_{0}^{T} x(t-\tau) h(\tau) d \tau \varphi(t) d t= \\
\int_{-\infty}^{\infty} x(t) \int_{0}^{T} h(\tau) \varphi(t+\tau) d \tau d t .
\end{gathered}
$$

Hence (13) implies (12). In the same way we can prove the converse. 


\section{Generalized Matched Filters}

The objective is to choose the function $h(\tau)$ so that it characterizes the detected signal in an optimal way. If we consider the case of the classical stationary processes $x(t)$ then the criterium of the system quality is maximizing the signal-to-noise ratio defined by the formula

$$
S / N=\frac{a_{o}^{2}\left(t_{0}\right)}{\sigma^{2}}
$$

where

$$
\sigma^{2}=E\left[|y(t)|^{2}\right]=\int_{0}^{T} \int_{0}^{T} h(u) B(u-v) \overline{h(v)} d v d u
$$

Here $B(u)$ is the correlation function of the process $x(t)$. Let us re-write the formula (18) with the help of the correlation functional $B(\varphi, \psi)$ of the generalized stationary process:

$$
\sigma^{2}=B(h, h)
$$

Then the formula (17) makes sense in the case of the generalized stationary process as well. We shall consider the following extremal problem.

Problem 1: Find $h(t) \in L^{2}(0, T)$ such that the signal-to-noise ratio $S / N$ has the greatest value.

The problems of the above type play an important role in communication theory [H00], [S97].

\section{V. $S_{J}$-Generalized Matched Filters}

We solve the problem for the case of $S_{J}$-generalized processes where $J=[0, T]$. In this case the equality

$$
\sigma^{2}=\left(S_{J} h, h\right)
$$

is valid. If $h(t)$ is a solution to the problem 1 then $c h(t)$ is a solution as well. Hence, without any loss of generality, we may assume that

$$
\int_{0}^{T} h(t) a\left(t_{0}-t\right) d t=1
$$

Problem 1 is equivalent to the following problem.

Problem 2: Find the minimum of the form $\left(S_{J} h, h\right)$ under the constraint (21) 
By solving the problem 2 we assume that there exists a function $f_{0}(t)$ which belongs to $L^{2}(0, T)$ and satisfies the relation

$$
S_{J} f_{0}=g_{0}, \quad \text { where } \quad g_{0}(t)=a\left(t_{0}-t\right) .
$$

Remark 2: If the operator $S_{J}$ is invertible then $f_{0}$ exists, and

$$
f_{0}=S_{J}^{-1} g_{0}
$$

Taking into account the equality (22) we can rewrite the condition (21) in the following form

$$
\left(h, S_{J} f_{0}\right)_{L^{2}}=\left(\sqrt{S_{J}} h, \sqrt{S_{J}} f_{0}\right)_{L^{2}}=1 .
$$

The latter and the Schwartz inequality imply $1 \leq\left(S_{J} h, h\right)_{L^{2}}\left(S_{J} f_{0}, f_{0}\right)_{L^{2}}$, i.e.,

$$
\left(S_{J} h, h\right) \geq \frac{1}{\left(S_{J} f_{0}, f_{0}\right)_{L^{2}}}=\frac{1}{\left(g_{0}, f_{0}\right)_{L^{2}}}=\nu_{\min } .
$$

Equality in (25) takes place if and only if

$$
\sqrt{S_{J}} h=\beta \sqrt{S_{J}} f_{0}
$$

Hence we obtain the minimal value

$$
\nu_{\min }=\frac{1}{\left(g_{0}, f_{0}\right)_{L^{2}}}
$$

when

$$
h=\beta f_{0} .
$$

Now, in view of the condition (21) we have

$$
\beta=\frac{1}{\left(g_{0}, f_{0}\right)_{L^{2}}} .
$$

Thus, the solution to the problem 1 has the form

$$
h_{\text {opt }}=\frac{f_{0}}{\left(g_{0}, f_{0}\right)_{L^{2}}}, \quad \mu_{\max }=S / N=\left(g_{0}, f_{0}\right)_{L^{2}} .
$$

where $g_{0}$ and $f_{0}$ are defined by the formulas (22) and (23), respectively.

Theorem 1: If the operator $S_{J}$ is invertible then there always exist a unique solution of the form (30) with $f_{0}=S_{J}^{-1} g_{0}$, i.e.,

$$
h_{\text {opt }}=\frac{S_{J}^{-1} g_{0}}{\left(g_{0}, S_{J}^{-1} g_{0}\right)_{L^{2}}}, \quad \mu_{\max }=S / N=\left(g_{0}, S_{J}^{-1} g_{0}\right)_{L^{2}} .
$$

Proposition 2: If $\left(S_{J} h, h\right)>0$ when $h \neq 0$ then the problem 1 has at most one solution.

Example 2: White noise. Recall that in white noise (9) case $S_{J}=I$ and hence the solution

$$
h_{\text {opt }}=\frac{a\left(t_{0}-t\right)}{\int_{0}^{T}\left|a\left(t_{0}-t\right)\right|^{2} d t}, \quad \text { and } \quad \mu_{\max }=S / N=\int_{0}^{T}\left|a\left(t_{0}-t\right)\right|^{2} d t
$$

is unique. 


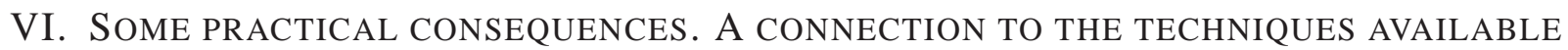
IN THE INTEGRAL EQUATIONS LITERATURE

The main focus of the sections II - V had mostly a theoretical nature. In this section we indicate that theorem 1 offers a novel technique allowing one to work out practical problems. Specifically:

- Filtering problems for classical stationary processes typically lead to non-invertible operators $S_{J}$, and to find the solution (30) one needs to solve (22).

In the case of generalized stationary processes the operator $S_{J}$ is often invertible and hence there are better formulas (31).

- Secondly, the operator $S_{J}$ in (7) and (8) can be seen as a new way of modelling colored noise. This model is useful since the existing integral equations literature already describes many particular examples on inverting $S_{J}$, either explicitly or numerically. Hence (31) solves the corresponding filtering problem.

Before providing two such examples we shall rewrite the operator $S_{J}$ of (8),

$$
S_{J} f=\frac{d}{d x} \int_{a}^{b} f(t) s(x-t) d t, \quad f(x) \in L^{2}(a, b)
$$

in a more familiar form. Let $J=[a, b]:=[0, w]$, and let $s(x)$ be a function differentiable when $x \neq 0$. Let

$$
s^{\prime}(x)=K(x) \in L^{2}(-w, w), \quad s(+0)-s(-0)=\mu .
$$

Then $S_{J}$ has the more familiar form

$$
S_{J} f=f(x) \mu+\int_{0}^{w} f(t) K(x-t) d t .
$$

The integral equations literature (see, e.g., [GS72], [S96]) contains results on the inversion of the operator $S_{J}$ of the form (32). The following theorem is well-known.

Theorem 2: (Gohberg-Semencul) Let the operator $S_{J}$ has the form (32) with $k(x) \in L(-w, w)$. If there are two functions $\gamma_{ \pm}(x) \in L(0, w)$ such that

$$
S_{J} \gamma_{+}(x)=k(x), \quad S_{J} \gamma_{-}(x)=k(x-w)
$$

then $S_{[0, w]}$ is invertible in $L^{p}(0, w)(p \geq 1)$ and

$$
S_{J}^{-1} f=f(x)+\int_{0}^{w} f(t) \gamma(x, t) d t
$$

where $\gamma(x, t)$ is given by (35). 


$$
\gamma(x, t)=\left\{\begin{array}{cccc}
-\gamma_{+}(x-t)- & \int_{t}^{w+t-x} & {\left[\gamma_{-}(w-s) \gamma_{+}(s+x-t)-\gamma_{+}(w-s) \gamma_{-}(s+x-t)\right] d s} & x>t \\
-\gamma_{-}(x-t)- & \int_{t}^{w} & {\left[\gamma_{-}(w-s) \gamma_{+}(s+x-t)-\gamma_{+}(w-s) \gamma_{-}(s+x-t)\right] d s} & x<t
\end{array}\right.
$$

The latter result leads to a number of special cases when the operator $S_{J}$ can be explicitly inverted and hence the formulas (31) solve the matching filter problem in these cases. We provide two such examples next.

Example 3: Colored noise approximated by rational functions. The exponential kernel. Let us consider colored noise approximated by a combination of rational functions with the fixed poles $\left\{ \pm i \alpha_{m}\right\}$,

$$
f(t)=\sum_{m=1}^{N} \gamma_{m} \frac{1}{t^{2}+\alpha_{m}^{2}}, \quad \alpha_{m}>0, \quad \gamma_{m}>0
$$

Let us use its Fourier transform

$$
K(x)=\sum_{m=1}^{N} \beta_{m} e^{-\alpha_{m}|x|}, \quad \beta_{j}=\frac{\pi}{\alpha_{m}} \gamma_{m}
$$

to define the operator $S_{J}$ via (32).

Solution to the filtering problem. The situation is exactly the one captured theorem 2 where the operator (32) has the special kernel (36). A procedure to solve (33), and hence to find the inverse of $S_{J}$ is obtained next.

Theorem 3: (Procedure) Let $S_{J}$ be given by (32) and its kernel $K(x)$ has the special form (36). Then $S_{J}^{-1}$ is given by the formula (34), (35), (33), where

$$
\gamma_{+}(x)=-\gamma(x, 0), \quad \gamma_{-}(x)=-\gamma(w-x, 0)
$$

Here

$$
\gamma(x, 0)=G(x)\left[\begin{array}{c}
F_{1} \\
F_{2}
\end{array}\right]^{-1} B
$$

where a $1 \times 2 N$ row $G(x), N \times 2 N$ matrices $F_{1}, F_{2}$ and a $2 N \times 1$ column $B$ are defined by

$$
\begin{aligned}
& G(x)=\left[\begin{array}{llll}
e^{\nu_{1} x} & e^{\nu_{2} x} & \cdots & e^{\nu_{2 N} x}
\end{array}\right], \quad F_{1}=\left[\frac{1}{\alpha_{i}+\nu_{k}}\right]_{1 \leq i \leq N, 1 \leq k \leq 2 N},
\end{aligned}
$$

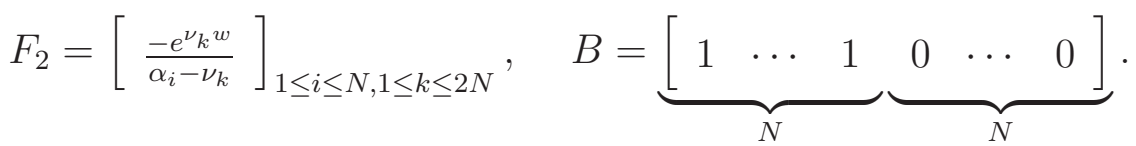

The numbers $\left\{\nu_{k}\right\}$ are the roots (we assume them to be pairwise different) of the polynomial

$$
Q(z)=P(z)-2 \sum_{m=1}^{N} \delta_{m} \sum_{s=1}^{m} \alpha^{2(m-s)} \sum_{k=1}^{N} \alpha_{k}^{2 s-1} \beta_{k}
$$


where the numbers $\left\{\delta_{k}\right\}$ are the coefficients of the polynomial

$$
P(z)=\sum_{m=1}^{N}\left(z^{2}-\alpha_{m}^{2}\right)=\sum_{m=1}^{N} \delta_{m} z^{2} m
$$

Hence, in this important case, the formulas (31) allow us to find the explicit solution $h_{\text {opt }}$ and $\mu_{\max }$ to the problem 1 via plugging in the formula (34), (35) together with (37) (38) that can be found using the methods of the integral equations theory.

Example 4: Positive frequencies white noise (PF-white noise). Recall (cf with the example 2) that the white noise case corresponds to $S_{J}=I$. The kernel $s(t)$ of such $S_{J}=I$ in (8) is the delta function which is the Fourier transform of $f_{W}(z)=1$ (i.e. equal intensity at all frequencies). Similarly, $f_{P W}(z)=\left\{\begin{array}{ll}1 & z \geq 0 \\ 0 & z<0\end{array}\right.$ represents the equal intensity at all positive frequencies and the zero intensity at the negative frequencies (hence the name PF-white noise). Then

$$
f(z)=f_{P W}(z)+(D-1) f_{W}(z), \quad D \geq 1
$$

represents a mix of white and PF-white noises. Let us now use the Fourier transform $s(t)$ of $f(z)$ in (41) as a kernel to define the operator $S_{J}$ via (8) (cf. with (32)), i.e.,

$$
S_{J} f=f D+\frac{j}{\pi} f_{0}^{T} \frac{f(t)}{x-t} d t, \quad f \in L_{2}[0, T],
$$

where $f_{0}^{T}$ is the Cauchy Principal Value integral, and $D \geq 1$.

Solution to the filtering problem. In the case of a pure PF-white noise (i.e., when $D=1$ ) the operator $S_{J}(42)$ is not invertible and (30) should be used. In the case $D>1$ the operator is invertible. Moreover, the following result is implicit in the literature. We provide its direct transparent proof of it in the appendix.

Theorem 4: (Explicit formula) If $D>1$ then $S_{J}$ is positive definite and invertible with

$$
S_{J}^{-1} f=f(x) D_{1}-\beta \frac{j}{\pi} f_{0}^{T}\left(\frac{t}{T-y}\right)^{j \alpha}\left(\frac{x}{T-x}\right)^{-j \alpha} \frac{f(t)}{x-t} d t
$$

where

$$
D_{1}=\frac{D}{D^{2}-1}, \quad \beta=\frac{1}{D^{2}-1},
$$

and the number $\alpha$ is obtained from

$$
\cosh \alpha \pi=D \sinh \alpha \pi \text {. }
$$

Hence, in this important case, the formulas (31) allow us to find the explicit solution $h_{\text {opt }}$ and $\mu_{\max }$ to the problem 1 via plugging in the formula (43) that is implicit the integral equations literature. 


\section{Appendix. Proof of TheOREM 3}

By comparing (32) and (34) we obtain

$$
\gamma(x, t)+K(x, t)+\int_{0}^{w} K(x, u) \gamma(u, t) d u=0
$$

and when $t=0$

$$
\gamma(x, 0)+K(x, 0)+\int_{0}^{w} K(x, u) \gamma(u, 0) d u=0 .
$$

Let us now apply to the both sides of (47) the operator $P(D)$, where $D$ is an operator of differentiation with respect to $x$, and $P(z)$ is a polynomial given in (40). As a result we obtain

$$
Q(D) \gamma(x, 0)=0
$$

where the polynomial $Q(z)$ is given by (39). The relation (48) implies

$$
\gamma(x, 0)=\sum_{m=1}^{2 N} c_{m} e^{\nu_{m} x}
$$

Now, (49) and (47) yield (38) Finally, using (38) we solve (33) and obtain (37).

\section{Appendix. Proof of TheOREM 4}

It is well-known [M77] that the operator

$$
V f=\frac{j}{\pi} f_{0}^{T} \frac{f(t)}{x-t} d t
$$

is a contraction: $\|V\|<1$. Hence the operator $S_{J}$ in (42) is nonnegative if $D \geq 1$. If $D>1$ then $S_{J}$ is invertible. A similar argument applied to the operator $T_{J}$ defined by the right hand side ${ }^{1}$ of (43) implies that if $D>1$ then $T_{J}$ is bounded, positive definite and invertible.

Let us check if $S_{J} T_{J}=I$. To this end let us compute

$$
\begin{gathered}
S_{J} T_{J} f=D D_{1} f+\frac{i D_{1}}{\pi} f_{0}^{T} \frac{f(t)}{x-t} d t-\beta \frac{i}{\pi} D f_{0}^{T}\left(\frac{t}{T-t}\right)^{i \alpha}\left(\frac{x}{T-x}\right)^{-i \alpha} \frac{f(t)}{x-t} d t+ \\
\frac{\beta}{\pi^{2}} f_{0}^{T}\left(\frac{x}{T-x}\right)^{-i \alpha}\left(\frac{t}{T-t}\right)^{i \alpha} \frac{1}{x-t} \cdot f_{0}^{T} \frac{f(u)}{t-u} d u .
\end{gathered}
$$

According to the Poincare-Bertran formula [M77]

$$
f_{0}^{T}\left(\frac{t}{T-t}\right)^{i \alpha} \frac{1}{x-t} f_{0}^{T} \frac{f(u)}{t-u} d u=f_{0}^{T} f(u) f_{0}^{T}\left(\frac{t}{T-t}\right)^{i \alpha} \frac{1}{(x-t)(t-u)} d t d u-\pi^{2} f(x)\left(\frac{x}{T-x}\right)^{i \alpha} .
$$

\footnotetext{
${ }^{1}$ Let us denote by $T_{J}$ a candidate for the inverse defined by the right hand side of (43).
} 
Denoting $W(z)=e^{-i \alpha \int_{0}^{T} \frac{d t}{t-z}}$ we see that it admits a representation

$$
W(z)=1+\frac{1}{2 \pi} \int_{0}^{T}\left[W_{+}(\sigma)-W_{-}(\sigma)\right] \frac{1}{\sigma-z} d \sigma
$$

where

$$
W_{ \pm}(\sigma)=\lim _{\tau \rightarrow \pm 0} W(\sigma+i \tau)=\left(\frac{\sigma}{T-\sigma}\right)^{i \alpha} e^{ \pm \pi \alpha}, \quad \sigma=\bar{\sigma}, \alpha=\bar{\alpha} .
$$

Now, the Sohotsky-Plemelj formula and (52) imply

$$
W_{+}(s)-W_{-}(s)=2+\frac{1}{\pi i} \int_{0}^{T} \frac{W_{+}(\sigma)-W_{-}(\sigma)}{\sigma-s} d \sigma=2+\frac{1}{\pi i} f_{0}^{T}\left(\frac{\sigma}{T-\sigma}\right)^{i \alpha}\left(e^{\pi \alpha}-e^{-\pi \alpha}\right) \frac{1}{\sigma-s} d \sigma .
$$

From here we obtain

$$
f_{0}^{T}\left(\frac{t}{T-t}\right)^{i \alpha} \frac{1}{(x-t)(t-u)}=\frac{\pi i}{u-x} \frac{\cosh \pi \alpha}{\sinh \pi \alpha}\left[\left(\frac{u}{T-u}\right)^{i \alpha}-\left(\frac{x}{T-x}\right)^{i \alpha}\right] .
$$

Finally, comparing of (50), (51) and (54), and using (44) and (45) we arrive at the desired identity

$$
S_{J} T_{J}=I
$$

\section{REFERENCES}

[L68] B.R.Levin, Theoretical Foundations of Statistical Radio Engineering, Moskow, 1968.

[GS72] I. Gohberg and A.Semencul, On the inversion of finite Toeplitz matrices and their continual analogues, Math. Issled. 7:2, 201-223. (In Russian.)

[GF74] I. Gohberg and I. A. Feldman, Convolution equations and projection methods for their solution, Transl. Math. Monographs, Providence, Rhode Island, 1974.

[H00] S.Haykin, Communications Systems, Wiley, 4th edition, 2000.

[LM04] Nadav Levanon, Eli Mozeson, Radar Signals, Wiley, 2004.

[M65] D. Middleton, Topics in Communication Theory, McGraw-Hill, 1965.

[M77] N.I. Muskhelishvili, Singular Integral Equations, Aspen Publishers Inc, 1977.

[S96] L.A.Sakhnovich, Integral Equations with Difference Kernels on Finite Intervals, Operator Theory Series, v.84, Birkhauser Verlag, 1996.

[S97] L.A.Sakhnovich, Interpolation Theory and its Applications, Kluwer Academic Publications, 1997.

[VG61] N.Y.Vilenkin and I.M.Gelfand, Some Applications of Harmonic Analysis, 1961.

[W49] N.Wiener, Extrapolation, Interpolation and Smoothing of Stationary Time Series, Cambridge, 1949.

[ZR50] L.A.Zadeh and J.R.Ragazzini, An extension of Wiener's Theory of Prediction, J.Appl.Physics, 1950, 21:7, 645-655. 